\title{
An Integrated Systems Model for Heavy Ion Drivers
}

\author{
W. R. Meier \\ R. O. Bangerter \\ A. Faltens \\ This paper was prepared for submittal to the \\ 12th International Symposium on Heavy Ion Fusion \\ Heidelberg, Germany \\ September 24-27, 1997
}

September 2, 1998

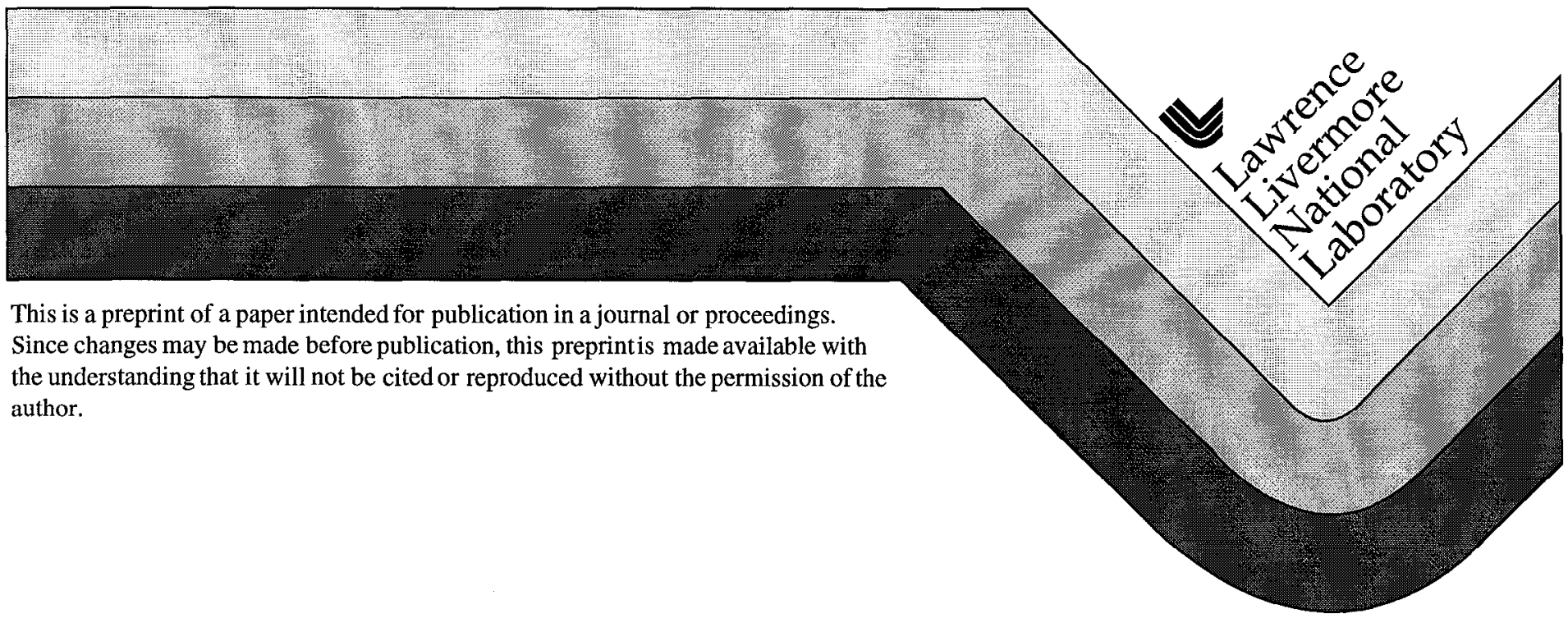




\section{DISCLAIMER}

This document was prepared as an account of work sponsored by an agency of the United States Government. Neither the United States Government nor the University of California nor any of their employees, makes any warranty, express or implied, or assumes any legal liability or responsibility for the accuracy, completeness, or usefulness of any information, apparatus, product, or process disclosed, or represents that its use would not infringe privately owned rights. Reference herein to any specific commercial product, process, or service by trade name, trademark, manufacturer, or otherwise, does not necessarily constitute or imply its endorsement, recommendation, or favoring by the United States Government or the University of California. The views and opinions of authors expressed herein do not necessarily state or reflect those of the United States Government or the University of California, and shall not be used for advertising or product endorsement purposes. 


\title{
Paper: SO07
}

\section{An Integrated Systems Model for Heavy Ion Drivers*}

\author{
W.R. Meier \\ Lawrence Livermore National Laboratory, Livermore, CA 94551, USA \\ R.O. Bangerter and A. Faltens \\ E.O. Lawrence Berkeley National Laboratory, Berkeley, CA. 94720, USA
}

\begin{abstract}
A source-to-target computer model for an induction linac driver for heavy ion fusion has been developed and used to define a reference case driver that meets the requirements of one current target design. Key features of the model are discussed, and the design parameters of the reference case design are described. Examples of the systems analyses leading to the point design are given, and directions for future work are noted.
\end{abstract}

Key Words: heavy ion, inertial confinement, fusion, driver, accelerator, systems model

PACS: 28.52.-s Fusion reactors

29.17.+w Electrostatic, collective, and linear accelerators

* Work performed under the auspices of the U.S. Department of Energy by Lawrence Livermore National Laboratory (Contract No. W-7405-Eng-48) and E.O. Lawrence Berkeley National Laboratory (Contract No. DE-AC03-76SF00098).

Send proofs to:

Wayne R. Meier

Lawrence Livermore National Laboratory

P.O. Box 808, L-481

Livermore, CA 94551

USA

Fax: $\quad 510-422-7390$

Phone: $510-422-8536$

E-mail: wmeier@llnl.gov 


\section{Introduction}

The heavy ion fusion programs at Lawrence Livermore National Laboratory and E.O. Lawrence Berkeley National Laboratory have been collaborating on the development of a computer model for systems analysis of heavy ion drivers based on induction linac technology. Work to date has focused on drivers for future heavy ion fusion (HIF) power plants, but the tools being developed will also be used to evaluate candidates for more near-term experimental facilities. Previous systems modeling and conceptual design studies for HIF drivers can be found in [1-4]. The model described here is an integrated source-to-target model that includes the key interdependencies of the major subsystems in terms of cost, performance and constraints. In this paper, we give a brief description of the general features of the model, including some example parametric studies, present the reference case design parameters, and conclude with the results of some cost sensitivity studies and directions for future work.

\section{Model description and example parameter studies}

\subsection{General features}

The computer model is written using a commercial scientific/engineering software package called Mathcad [5] that incorporates text and graphics in an easily readable and user-friendly format. The reference case induction linac driver architecture includes a multi-beam injector feeding an electrostatic-focusing accelerator section, four-to-one beam merging and transition to a magnetic-focusing accelerator section at some higher energy (e.g., $\sim 100 \mathrm{MeV}$ ). The main accelerator is followed by final transport and final focus sections. The final transport section 
provides the distance needed for drift compression (to get the required pulse length on target) and re-alignment of the beams for two-sided illumination. The final focus section includes the final focus quadrupoles that are used to first expand the beam radially and then focus it to a small spot on target. Calculation of the spot size on target is included.

The heart of the model is the set of transport, component sizing, and cost scaling algorithms that describe the components in each section of the driver. Numerous inputs, assumptions and constraints for the models of the various components are defined throughout the program. Typical assumptions are cost scaling dependencies and component unit costs (e.g., $\$ / \mathrm{kg}$ of various materials). Examples of constraints include the source current density limit $\left(\mathrm{A} / \mathrm{m}^{2}\right)$ and the acceleration gradient limit $(\mathrm{V} / \mathrm{m})$ in the accelerator. A key input is the specification of bcam characteristics that the driver must deliver to the target. Table 1 gives the target requirements for one current HIF target design [6]; other target designs will be investigated in future work. Note that the target requires a prepulse with a lower ion energy $(3 \mathrm{GeV})$ than the main pulse $(4 \mathrm{GeV})$.

\subsection{Injector}

The multi-beam injector delivers ions at $2 \mathrm{MeV}$ simultaneously to each beam channel in the low-energy, electrostatic-focusing section of the accelerator. Eventually, the injector voltage will be determined by comparing the cost of the injector as a function of voltage to the cost of induction acceleration at low voltage and picking the lowest cost approach. For now, we are assuming a $2 \mathrm{MeV}$ injector because that is our present experience. The total charge that must be transported is calculated from the energy on target and final voltages of the prepulse and main pulse beams. The initial current per beam at the exit of the injector is the charge per beam 
divided by the initial pulse duration, which is one of the design variables we optimize. In our model, the number of prepulse and main pulse beams is set so that each beam carries the same charge. In this way, the entire accelerator up to $3 \mathrm{GeV}$ is identical for prepulse and main pulse beams. The source radius for each beam depends on the achievable source current density $\left(\mathrm{A} / \mathrm{m}^{2}\right)$, which is a function of diode gap as described by the Child-Langmuir law and is constrained by emission limits for the particular ion source. Since for good optics the diode gap is proportional to the source radius, there is a unique combination of source radius, diode gap, and diode voltage that gives the required current per beam. For example, the reference case design with 192 beams at the low energy end has a source radius of $29 \mathrm{~mm}$, diode gap of $73 \mathrm{~mm}$, and a diode voltage of $\sim 300 \mathrm{kV}$, providing a current of 0.3 A per beam at a $30 \mu$ s pulse duration.

\subsection{Electrostatic focusing section}

Electrostatic quadrupoles are used at the low energy end of the accelerator, from the $2 \mathrm{MeV}$ injector energy to $\sim 100 \mathrm{MeV}$ in our reference case design. The general configuration of a multibeam array is illustrated in Fig. 1. (For simplicity and clarity, Fig. 1 shows a 32-beam array instead of the 192-beam array used as our reference design. The 192 beams will be configured in

a similar pattern.) The beam size depends on the current per beam coming from the injector. Electrode dimensions are scaled from the beam size and in turn determine the outer radius of the multi-beam array and the inner radius of the induction cores. Here we find the inner radius decreases with increasing number of beams and increasing initial pulse duration. For our reference case of 192 beams and $30 \mu \mathrm{s}$, the inner radius of the cores is $0.37 \mathrm{~m}$. 
The acceleration gradient increases from an initial value of $\sim 50 \mathrm{kV} / \mathrm{m}$ to $330 \mathrm{kV} / \mathrm{m}$ at $100 \mathrm{MeV}$; it is limited by the maximum allowable radial build of the cores, which is a user specified constraint ( $1.0 \mathrm{~m}$ in this case). This then determines the length of the accelerator required to achieve a given ion energy, which is $492 \mathrm{~m}$ to an ion energy of $100 \mathrm{MeV}$.

The cost of the electrostatic quadrupoles is broken down into six subsystems: electrodes, end plates, insulators, power supplies, vacuum vessel, and articulation. The cost of the accelerator modules (or cores) includes the cost of magnetic material, cell housing, structural support, and gap insulators. The electrostatic focusing section cost is the sum of the costs for quadrupoles, cores, pulsed power and the vacuum pumping systems. To this we add the cost of the injector to get the cost of the front end. Cost scaling relationships have been developed for all these subsystems.

Fig. 2 shows the cost of the front end as a function of the initial pulse duration. The costs have been normalized to the minimum, which occurs at $30 \mu \mathrm{s}$. The cost is strongly dominated by the cost of cores for pulse durations greater than $30 \mu \mathrm{s}$. At lower pulse durations, the injector cost rises rapidly due to the higher initial current per beam and correspondingly high source area requirements. The cost of the front end also decreases with increasing number of beams, but reaches diminishing returns beyond 150-200 beams. The front end cost of our 192-beam reference case design is only $10 \%$ higher than a 300 beam case.

\subsection{Magnetic focusing section}


At the transition from electrostatic to magnetic focusing, the multi-beam array undergoes a four-to-one beam merging, which gives 48 beams in the magnetic section. The transition energy is a design variable that is chosen to minimize the overall driver cost (including front end, magnetic focusing section, final transport and final focusing). As will be shown later, the cost is rather insensitive to this transition energy over 10 's of $\mathrm{MeV}$, with the optimum at $\sim 100 \mathrm{MeV}$.

As in the electrostatic section, all of the beams in the magnetic quadrupole section share common cores. Each quadrupole occupies a square unit cell, illustrated in Fig. 3, consisting of an inner vacuum pipe (bore) wall, a 0.5 -cm-thick layer of superinsulation, a support pipe wall on which the superconductor is wound, and regions (primarily the corners of the square) for superconductor and associated cooling. The entire package is surrounded by a structural metal box on the outer edge. The inner radius of the bore is scaled from the beam size, which depends on the current per beam and the magnetic field of the quadrupole. The thicknesses of the pipe walls and the outer box wall scale in proportion to the beam size and are set by stress considerations. One half of a unit cell thickness of conductor surrounds the entire array to provide a flux return path for the quadrupoles on the outer edge.

The overall radial dimension of the array determines the inner radius of the induction cores. Fig. 4 shows the core radius as a function of the quadrupole field at the winding, $\mathrm{B}_{\mathrm{W}}$, for several different initial pulse durations. The minimum inner radius occurs at $6 \mathrm{~T}$ in each case. At higher fields, extra space is needed for the conductor, and the quadrupole unit cell and overall array dimensions increase. While $6 \mathrm{~T}$ gives the smallest core radius, the minimum cost for the driver occurs at $\mathrm{B}_{\mathrm{W}}=4 \mathrm{~T}$. This illustrates the importance of an integrated model. 
As shown in Fig. 5, the acceleration gradient in the magnetic focusing section increases rapidly to $1 \mathrm{MV} / \mathrm{m}$ and then rises slowly to a maximum of nearly $2 \mathrm{MV} / \mathrm{m}$. As the acceleration gradient is increased, the core axial packing fraction is decreased to reduce capacitance and prevent breakdown. Once the ions reach $3 \mathrm{GeV}$, the array is split into two parallel multi-beam arrays, one for the prepulse ( 16 beams) and one for the main pulse ( 32 beams). As the main pulse beams continue to accelerate to $4 \mathrm{GeV}$, the prepulse beams are simply transported to the end of the accelerator.

\subsection{Final transport and focusing}

At the end of the accelerator, the prepulse and main pulse have pulse durations of 140 and $110 \mathrm{~ns}$, respectively. These must be shortened to 30 and $8 \mathrm{~ns}$, respectively, prior to focusing on target. A simple model is used to calculate the required velocity tilt and drift length to accomplish this [7]. Secondly, we determine the transport length needed simply to redirect the beams to a two-sided illumination geometry as a function of the field in the dipole bending magnets. The larger of the two distances sets the final transport length. The final transport cost includes the costs of quadrupoles, dipoles, and a vacuum system. In our reference case, a dipole field of 4.5 T gave the minimum cost and resulted in final transport length (from the end of the accelerator to the end of the final focus magnets) of $415 \mathrm{~m}$. Note that as the pulses are compressed, the current per beam rises rapidly, and the bore radius and quadrupole fields are increased in order to transport the higher currents. 
The final focus geometry is based on a simple point-to-point lens model consisting of four quadrupoles for each beam [8]. Magnet dimensions and costs scale with the beam size at the middle of the final quadrupole, which is equal to the product of the beam focusing half angle, $\theta_{\mathrm{f}}$, and the final focus length, $\mathrm{L}_{\mathrm{f}}$ (distance from the middle of the last quadrupole to the target). An allowance for neutron shielding inside the bore of the final focus quadrupoles is included, although more detailed work is needed on this aspect of the design. The inner radius of the bore is also constrained by voltage breakdown between the beam and bore wall, using a limit of $200 \mathrm{kV} / \mathrm{cm}$, which is adequate for short duration pulses.

\section{Reference case parameters}

Table 2 shows several parameters of the reference case design which delivers the required $5.9 \mathrm{MJ}$ to the target. The overall length of the accelerator is $3250 \mathrm{~m}$ plus an additional $415 \mathrm{~m}$ for the final transport and focusing. The current per beam increases from $0.3 \mathrm{~A}$ at the exit of the injector to $1.1 \mathrm{kA}$ for the prepulse and $4.2 \mathrm{kA}$ for the main pulse. The beam radius is less than $7 \mathrm{~mm}$ in both the electrostatic and magnetic focusing sections, and the bore radius is $\sim 13 \mathrm{~mm}$.

\section{Cost results}

\subsection{Cost breakdown}

The total direct cost of the 5.9 MJ reference case driver is $\sim \$ 1.4 \mathrm{~B}$. As indicated in Fig. 6 , the contributions to this total are: injector (2\%), electrostatic focusing section (10\%), magnetic 
focusing section (48\%), final transport (6\%), and final focus (3\%). We include an allowance for instrumentation and control (6\%) and installation and assembly (23\%). We note that these results are preliminary, and work continues on refining the cost scaling models and accelerator architecture in order to reduce this cost. If we examine the cost of the magnetic focusing section in more detail, we find that its cost is dominated by the cost of cores (40\%) and pulsed power (40\%). The quadrupoles and vacuum system account for $14 \%$ and $6 \%$, respectively.

\subsection{Cost sensitivity}

We have also examined the sensitivity of the driver cost to changes in reference point design variables and have found that the cost is relatively insensitive to parameter variations over a rather broad range. The driver cost is most sensitive to reducing the initial pulse duration $(+17 \%$ going to $15 \mu \mathrm{s})$ and reducing the number of beams ( $+13 \%$ for half as many beams). Changing the quadrupole field over the range of 2 to $6 \mathrm{~T}$ increases the cost by $5 \%$ or less. The transition energy from electrostatic to magnet focusing has very little impact over the range of 50 to $150 \mathrm{MeV}$.

\section{Future work}

This work is still in progress, and several things are planned for the future. First, we will conduct studies with the current architecture to find optimum designs and costs for different ions, ion energies and targets, including higher charge-to-mass ratio ions. We will work to improve the cost basis and cost scaling models, develop a better treatment of emittance growth along the accelerator, and provide more detail on the final focusing model. Technical solutions, such as 
using fewer, more powerful thyratrons for the pulsed power at the high energy end, will also be incorporated. Finally, we plan to evaluate other architectures (e.g., an all-magnetic front end, elimination of merging, replacing part of the magnetic section with a recirculating linac).

\section{Acknowledgments}

We would like to thank John Barnard, Debra Callahan-Miller, Alex Friedman, and Grant Logan of LLNL and Joe Kwan and Ed Lee of LBNL for their reviews of various parts of the model and useful suggestions.

\section{References}

[1]D.J. Dudziak, W.W. Saylor, and W.B. Herrmannsfeldt, "U.S. heavy-ion fusion systems assessment project overview," Fusion Technol., 13, (Feb. 1988) 207.

[2] D.S. Zuckerman, D.E. Driemeyer, L.M. Waganer, and D.J. Dudziak, "An induction linac driven heavy-ion-fusion systems model," Fusion Technol., 13 (Feb. 1988) 217.

[3] J. Hovingh, V.O. Brady, A. Faltens, and D. Keefe, and E.P. Lee, "Heavy-ion linear induction accelerators as drivers for inertial fusion power plants," Fusion Technol., 13 (Feb. 1988) 255.

[4] R.L. Bieri, M.J. Monsler, W.R. Meier, and L.Stewart, "Heavy-ion driver design and scaling," Fusion Technol., 21 (May 1992) 1583.

[5] Mathcad is a registered trademark of MathSoft, Inc., 101 Main St. Cambridge, MA 02142, USA, Phone: 800-628-4223, Ineternet: www.mathsoft.com.

[6] M. Tabak and D. Callahan-Miller, "Distributed radiator heavy ion target designs," these Proceedings.

[7] E.P. Lee, C. Fong, S. Mukherjee, and W. Thur, "Conceptual design of bend, compression and final focus components of ILSE," IEEE report CH2669-0/89/0000-0971 (1989). 
[8] E.P. Lee, "Heavy ion driven LMF design concept," LBNL report LBL-31248 revised (July 1995). 
Table 1

Target requirements

\begin{tabular}{lcc}
\hline & Prepulse & Main Pulse \\
\hline Ion mass, amu & 200 & 200 \\
Ion energy, GeV & 3.0 & 4.0 \\
Beam energy, MJ & 1.6 & 4.3 \\
Total charge, mC & 0.53 & 1.07 \\
Pulse duration on target, $\mathrm{ns}$ & 30 & 8 \\
Spot radius on target, $\mathrm{mm}$ & 2.7 & 2.7 \\
\hline
\end{tabular}

Table 2

Reference case design parameters

\begin{tabular}{|c|c|c|c|c|}
\hline \multicolumn{3}{|c|}{ Number of beams (electrostatic/magnetic) } & \multicolumn{2}{|c|}{$192 / 48$} \\
\hline \multicolumn{3}{|l|}{ Initial pulse duration, $\mu$ s } & \multicolumn{2}{|c|}{30} \\
\hline \multicolumn{3}{|c|}{ Transition to magnetic focusing, $\mathrm{MeV}$} & \multicolumn{2}{|c|}{100} \\
\hline \multicolumn{3}{|c|}{ Accelerator quadrupole field at winding, $T$} & \multicolumn{2}{|c|}{4.0} \\
\hline \multicolumn{3}{|c|}{ Final transport quad field at winding, $\mathrm{T}$} & \multicolumn{2}{|c|}{4.5} \\
\hline \multicolumn{3}{|l|}{ Final focus length, m } & \multicolumn{2}{|c|}{5.0} \\
\hline \multicolumn{3}{|c|}{ Beam focus half angle, mrad } & \multicolumn{2}{|c|}{10} \\
\hline & \multicolumn{4}{|c|}{ Along accelerator } \\
\hline & $\begin{array}{c}\text { Injector } \\
\text { Exit }\end{array}$ & $\begin{array}{l}\text { End of } \\
E Q F^{\mathrm{a}}\end{array}$ & $\begin{array}{l}\text { Pre- } \\
\text { pulse }\end{array}$ & $\begin{array}{l}\text { Main } \\
\text { Pulse }\end{array}$ \\
\hline Ion energy, GeV & 0.002 & 0.1 & 3.0 & 4.0 \\
\hline Pulse duration, $\mu \mathrm{s}$ & 30 & 4.2 & 0.14 & 0.11 \\
\hline Pulse length, m & 41.7 & 41.7 & 7.6 & 6.6 \\
\hline Beam current, $A$ & 0.3 & 2.0 & 230 & 310 \\
\hline Beam radius, mm & 6.6 & 6.6 & 6.9 & 6.9 \\
\hline Bore radius, mm & 12.6 & 12.6 & 12.9 & 12.9 \\
\hline Core inner radius, $\mathrm{m}$ & 0.37 & 0.37 & 0.29 & 0.25 \\
\hline Core build, m & 0.92 & 1.0 & 0.89 & 0.4 \\
\hline Accel. gradient, MV/m & 0.05 & 0.33 & 1.77 & 1.91 \\
\hline \multirow[t]{3}{*}{ Dist. from injector, $m$} & 0 & 490 & 2220 & 3250 \\
\hline & \multicolumn{4}{|c|}{ At last final focus quadrupole } \\
\hline & \multicolumn{2}{|c|}{ Prepulse } & \multicolumn{2}{|c|}{ Main Pulse } \\
\hline Pulse duration, ms & \multicolumn{2}{|c|}{30} & \multicolumn{2}{|c|}{8} \\
\hline Pulse length, m & \multicolumn{2}{|c|}{1.6} & \multicolumn{2}{|c|}{0.5} \\
\hline Beam current, A & \multicolumn{2}{|c|}{1110} & \multicolumn{2}{|c|}{4170} \\
\hline Beam radius, mm & \multicolumn{2}{|c|}{50} & \multicolumn{2}{|c|}{50} \\
\hline Bore radius, mm & \multicolumn{2}{|c|}{70} & \multicolumn{2}{|c|}{70} \\
\hline
\end{tabular}

${ }^{a_{E Q F}}$ = Electrostatic quadrupole focusing 


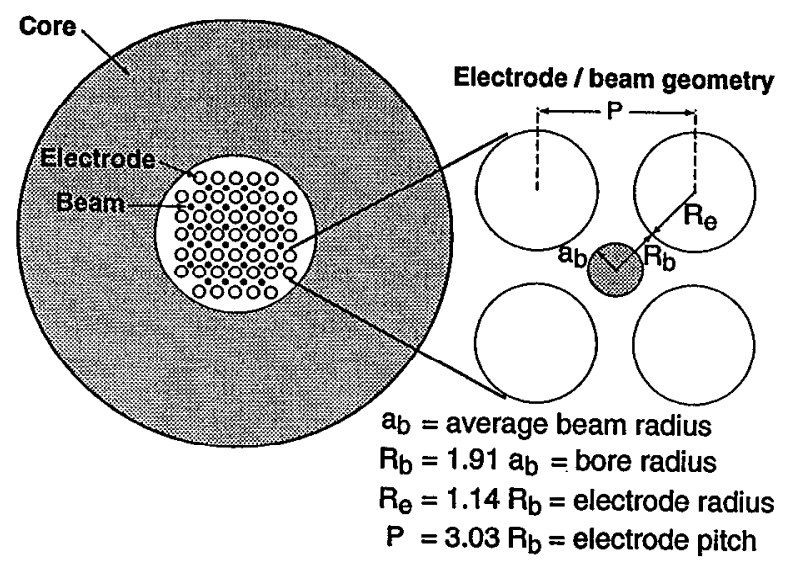

Fig. 1. Configuration of electrostatic quadrupole array. 


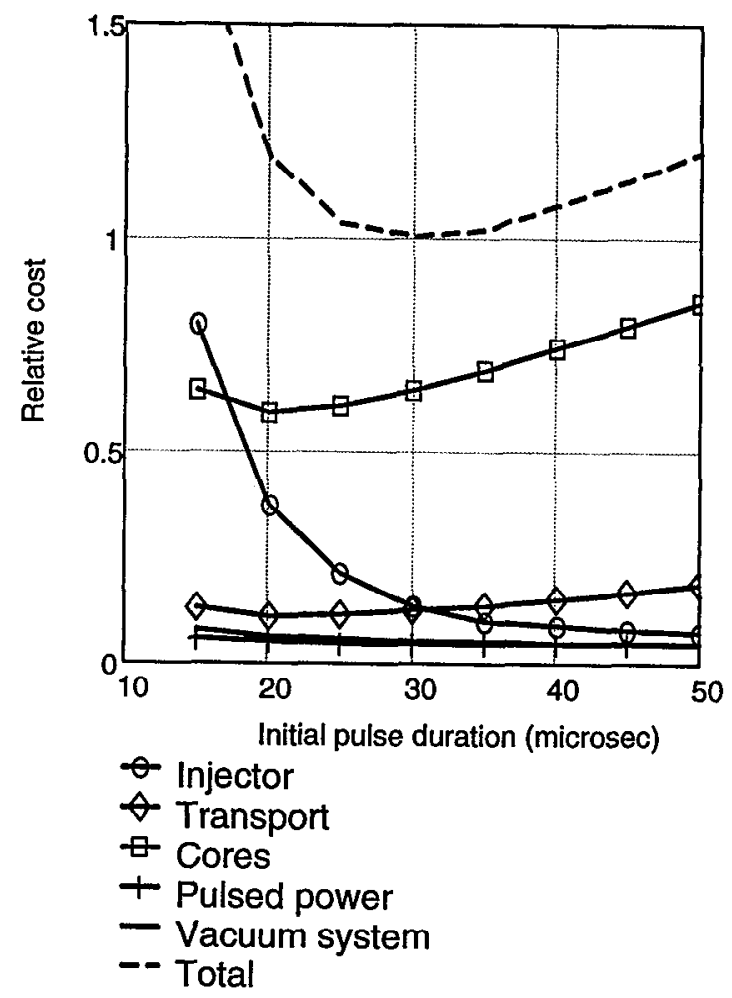

Fig. 2. Relative cost of front end (up to $100 \mathrm{MeV}$ ) as a function of initial pulse duration for 192 beam case. 


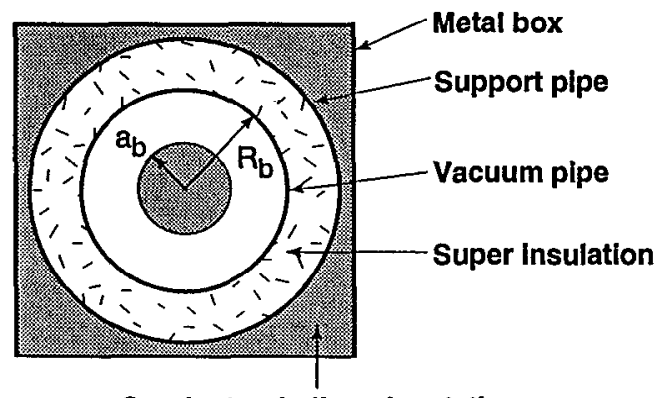

Conductor, helium, insulation

$a_{b}=$ average beam radius

$R_{b}=1.82 a_{b}=$ bore radius

Fig. 3. Configuration of magnetic quadrupoles. 


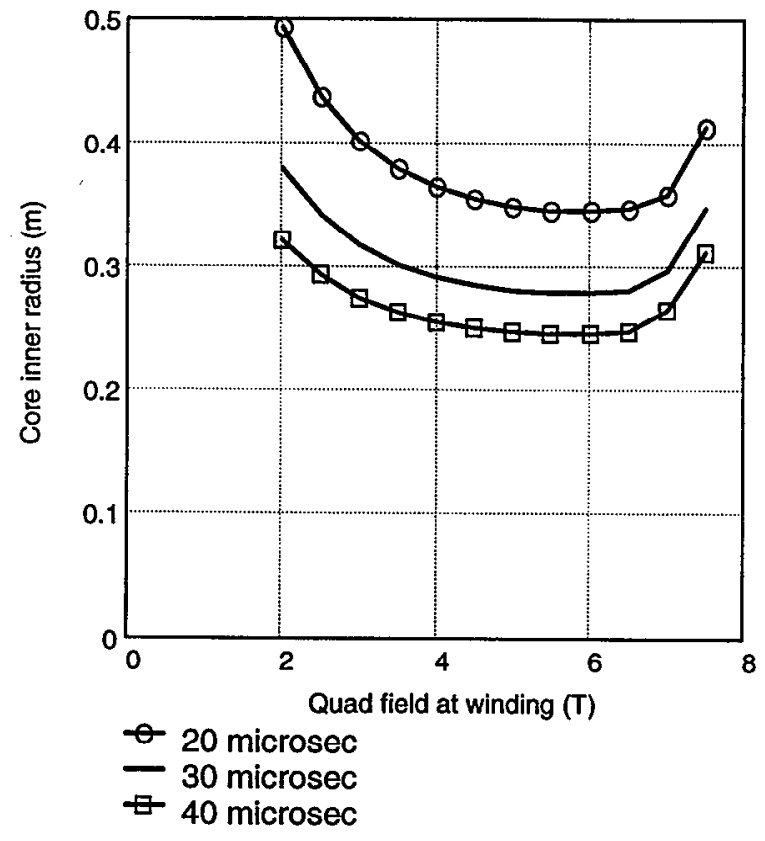

Fig. 4. Core inner radius as a function of the quadrupole field at the winding for initial pulse durations of 20,30 and $40 \mu \mathrm{s}$. 


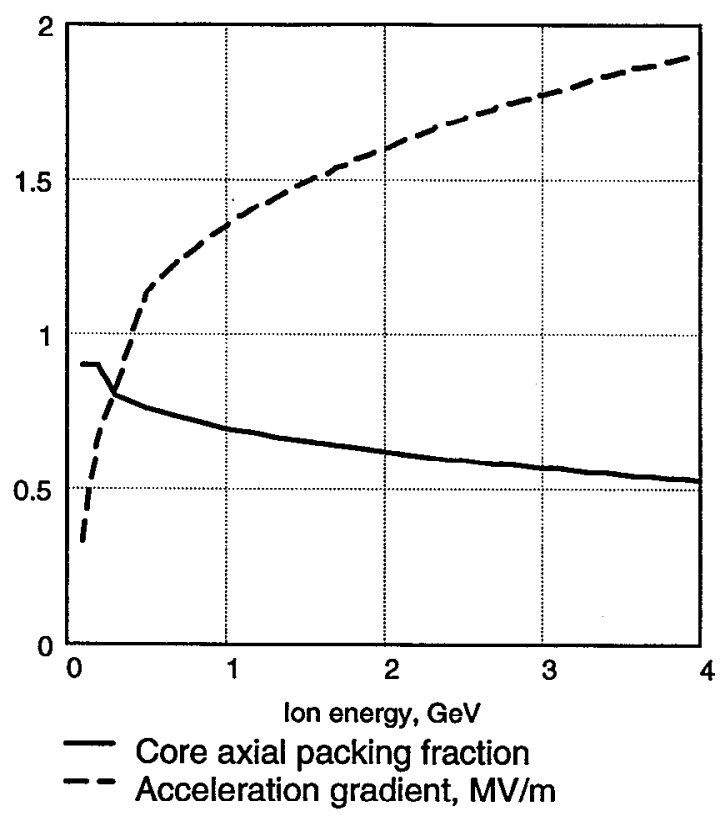

Fig. 5. Acceleration gradient and core axial packing fraction as a function of ion energy in the magnetic focusing section. 


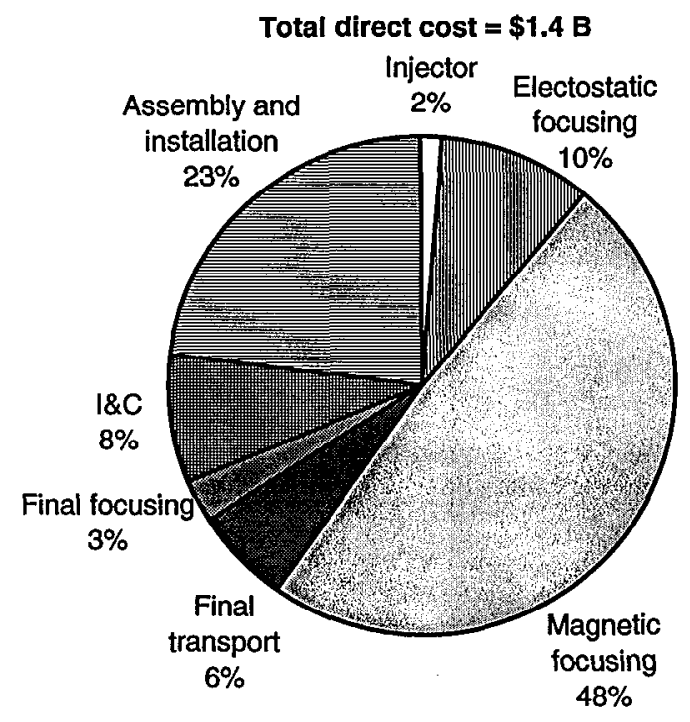

Fig. 6. Cost breakdown for reference case design. 


\section{List of figure captions}

Fig. 1. Configuration of electrostatic quadrupole array.

Fig. 2. Relative cost of front end (up to $100 \mathrm{MeV}$ ) as a function of initial pulse duration for 192 beam case.

Fig. 3. Configuration of magnetic quadrupoles.

Fig. 4. Core inner radius as a function of the quadrupole field at the winding for initial pulse durations of 20,30 and $40 \mu$ s.

Fig. 5. Acceleration gradient and core axial packing fraction as a function of ion energy in the magnetic focusing section.

Fig. 6. Cost breakdown for reference case design. 